\title{
Diaphragmatic hernia after Ivor Lewis esophagectomy for cancer: a retrospective analysis of risk factors and post-repair outcomes
}

\author{
Francesco Puccetti ${ }^{1}$, Andrea Cossu ${ }^{1}$, Paolo Parise ${ }^{1}$, Lavinia Barbieri ${ }^{1}$, Ugo Elmore ${ }^{1}$, Agnese Carresi ${ }^{1}$, \\ Stefano De Pascale ${ }^{2}$, Uberto Fumagalli Romario ${ }^{2}$, Riccardo Rosati ${ }^{1}$ \\ ${ }^{1}$ Department of Gastrointestinal Surgery, San Raffaele Hospital IRCCS, Milan, Italy; ${ }^{2}$ Digestive Surgery Unit, European Institute of Oncology \\ IRCCS, Milan, Italy \\ Contributions: (I) Conception and design: F Puccetti, A Cossu, P Parise, R Rosati; (II) Administrative support: F Puccetti, P Parise, U Elmore, U \\ Fumagalli Romario, R Rosati; (III) Provision of study materials or patients: P Parise, U Elmore, U Fumagalli Romario, R Rosati; (IV) Collection \\ and assembly of data: F Puccetti, A Cossu, P Parise, L Barbieri, A Carresi, S De Pascale; (V) Data analysis and interpretation: F Puccetti, A Cossu, \\ P Parise, U Elmore, S De Pascale, U Fumagalli Romario, R Rosati; (VI) Manuscript writing: All authors; (VII) Final approval of manuscript: All \\ authors.
}

Correspondence to: Francesco Puccetti, MD. Via Olgettina 60, 20132 Milan, Italy. Email: puccetti.francesco@hsr.it.

Background: Esophageal cancer surgery has historically been associated with high levels of postoperative morbidity and mortality. Post-esophagectomy diaphragmatic hernia (PEDH) represents a potentially lifethreatening surgical complication, with incidence and risk factors not clearly demonstrated. This study evaluates presenting characteristics and repair outcomes in PEDH after Ivor Lewis esophagectomy for cancer.

Methods: All consecutive patients who underwent esophageal cancer surgery between March 1997 and April 2018 at two high-volume centers were included. The patients underwent Ivor Lewis esophagectomy and were managed according to a standardized follow-up care plan. The primary outcomes included PEDH incidence, risk factor identification, and surgical results after hernia repair. Patient characteristics and perioperative data were collected and a multivariate analysis was performed to identify risk factors for PEDH.

Results: A total of 414 patients were enrolled and $22(5.3 \%)$ were diagnosed with PEDH during a median follow-up period of 16 (range, 6-177) months. All patients underwent surgical repair and 16 (73\%) required treatment within 24 hours. PEDH repair was mainly performed through a laparoscopic approach (77.3\%), with an overall postoperative morbidity of $22.7 \%$ and one mortality case. The median length of hospital stay was 6 (range, 2-95) days, and no early recurrences were observed, although three (13.6\%) cases relapsed over a median follow-up of 10.1 months after hernia repair. Univariate analysis demonstrated a statistically significant association between $\mathrm{PEDH}$ and neoadjuvant chemoradiotherapy $(\mathrm{P}=0.016)$, pathological complete response $(\mathrm{P}=0.001)$, and lymph node harvest $(\mathrm{P}=0.024)$. On the other hand, multivariate analysis identified pathological complete response [3.616 (1.384-9.449), P=0.009] and lymph node harvest [3.029 (1.140-8.049), $\mathrm{P}=0.026]$ as the independent risk factors for developing $\mathrm{PEDH}$.

Conclusions: PEDH represents a relevant surgical complication after Ivor Lewis esophagectomy for cancer, including a 5.3\% incidence and requiring surgical repair. Pathological complete response and lymph node harvest were found to be independent risk factors for PEDH, independently of the esophagectomy technique.

Keywords: Hiatal hernia; esophagectomy; esophageal cancer; postoperative complications

Submitted May 20, 2020. Accepted for publication Oct 15, 2020.

doi: $10.21037 /$ jtd-20-1974

View this article at: http://dx.doi.org/10.21037/jtd-20-1974

(c) Journal of Thoracic Disease. All rights reserved. 


\section{Introduction}

Esophagectomy represents the mainstay component of multimodal treatment for esophageal cancer, although it has been historically associated with significant morbidity and mortality rates (1). Post-esophagectomy diaphragmatic hernia $(\mathrm{PEDH})$ is considered as a potentially lifethreatening complication requiring surgical repair to prevent severe conditions such as conduit obstruction or perforation and respiratory failure $(2,3)$.

Although several studies have previously analyzed the occurrence of diaphragmatic hernia after esophageal surgery, no consensus has been reached concerning both incidence and risk factor identification due to the large variety of study designs and selection criteria. The current literature has provided individual institution analysis reporting estimated incidence rates widely ranging from $0.3 \%$ to $19 \%$ according to study characteristics, such as operative technique, underlying esophageal disease, and length of postoperative follow-up (3-5). These limitations have not allowed reaching clear evidence regarding either pathological mechanisms or predictors of PEDH. In particular, the role of surgical technique has been debated since previous studies advocated that minimally invasive esophagectomy is associated with a higher risk of transhiatal herniation up to $26 \%$ (6-9). However, these results remain controversial, while larger evidence has supported the effectiveness of keyhole surgery in providing long-term survival benefits as well as reduced postoperative morbidity and length of hospital stay (10-13).

The occurrence rate of $\mathrm{PEDH}$ has been an increasing issue due to both diagnostic and therapeutic advances of the multimodal approach, which has improved the overall survival of esophageal cancer (14). On the other hand, the impact of esophagectomy on cancer patients' survival and quality of life remains considerable, and PEDH has been demonstrated to be a surgical complication potentially affecting both aspects.

Therefore, this study aims to determine the incidence and risk factors of postoperative diaphragmatic herniation after Ivor Lewis esophagectomy and to analyze surgical outcomes following hernia repair. We present the following article in accordance with the STROBE Statement reporting checklist (available at http://dx.doi.org/10.21037/jtd-20-1974).

\section{Methods}

\section{Study design}

This retrospective study analyzed all patients who underwent esophageal cancer surgery at two high-volume centers between March 1997 and April 2018. The inclusion criteria for patient selection were histologic diagnosis of esophageal cancer and Ivor Lewis esophagectomy with twofield lymphadenectomy and intra-thoracic anastomosis, independent of surgical technique and neoadjuvant therapy. After esophagectomy, all patients were managed according to a standardized follow-up care plan. This standardized 5 -year follow-up was similar between institutions and involved periodic clinical examinations and investigations, including both CT scan and endoscopy at 8, 12, 24, 36, 48 , and 60 months after surgery. PEDH was defined as partial or entire migration of any abdominal organ, other than the gastric conduit, through the esophageal hiatus. All radiologic findings consistent with PEDH were independently evaluated by two radiologists reporting a detailed description of dimensions, involved organs, and visceral perfusion. All radiologically confirmed PEDHs which involved more than one abdominal organ were defined as multi-visceral, whereas those occurring within 90 days after esophagectomy were classified as early hernia. Moreover, PEDHs showing clinical or radiological indications of gastrointestinal volvulus, ischaemia, sepsis, or respiratory dysfunction were classified as complicated hernia. However, all PEDH patients were scheduled for elective repair to prevent more severe complications related to trans-hiatal herniation, while those presenting with acute symptoms underwent surgical repair within 24 hours. The primary outcomes included the estimation of PEDH incidence and the identification of risk factors among potential predictors, such as patient, oncologic disease, and peri-esophagectomy characteristics, and repair surgery and postoperative results were also primary endpoints. The present study was conducted in accordance with the Declaration of Helsinki (as revised in 2013). According to the national law regulating research ethics (resolution March 1, 2012, Gazzetta Ufficiale n.72 of March 26, 2012) and due to the retrospective design of the study, the ethical approval and informed consent have been waived since all analyzed data were collected as part of routine diagnosis and treatment, which were consistent with the current standard of care.

\section{Techniques of esophagectomy}

All consecutive patients underwent curative Ivor Lewis esophagectomy involving gastric conduit reconstruction. The abdominal phase started with the Kocher manoeuvre, 
followed by complete gastric mobilization. Abdominal lymphadenectomy included dissection of the gastric lesser curvature, hepatic artery, and celiac stations. The gastric conduit was intra-abdominally shaped and the proximal stomach was resected along with the regional lymph nodes. The dissection of the lower esophagus was initially performed through a trans-hiatal approach, and, in case of a tight diaphragmatic hiatus, a partial resection of the right crus was occasionally performed (15). The following thoracic phase involved the resection of the azygos vein and mobilization of the whole esophagus up to the upper third. In thoracic lymphadenectomy, the main stations (bronchial, subcarinal, middle and lower esophageal, paratracheal, and inter-aortocaval nodes) were removed, with additional dissection of those surrounding the upper third and recurrent laryngeal nerve in case of middle-third esophageal cancer. The gastric conduit was pulled into the chest, and a mechanical esophagogastric anastomosis was performed. Furthermore, Ivor Lewis esophagectomy was performed using three different techniques according to disease characteristics and the surgeon's preference: totally open incision, hybrid approach with abdominal laparoscopy plus right thoracotomy, and total minimally invasive esophagectomy.

\section{Techniques of hernia repair}

Minimally invasive repair was the treatment of choice, and a chest tube was placed prior to the induction of the pneumoperitoneum to avoid hypertensive pneumothorax. An intra-abdominal exploration was performed to evaluate the diaphragmatic defect and exclude possible signs of ischaemic injury or perforation of the migrated content. The hiatal hernia was reduced into the abdominal cavity by means of extended and accurate adhesiolysis, carefully avoiding damaging the gastroepiploic vessels. The repair was standardly performed by cruroplasty with a thick, polyester, non-absorbable suture, whereas the crural mesh was exclusively used in case of residual weakness of diaphragmatic closure.

\section{Data recording and statistical analysis}

All data concerning patient characteristics, surgical treatment, and multimodal therapy were prospectively collected into an institutional clinical database by a dedicated data manager. All PEDH cases were identified and additional data regarding clinical presentation and surgical repair were extracted. Postoperative complications reporting a Clavien-Dindo score $\geq 3$ a were defined as severe, whereas early mortality included all deaths occurring within 90 days $(16,17)$. Fisher's exact or Pearson's $\chi^{2}$ test and Mann-Whitney $U$ test were appropriately used in evaluating categorical and continuous variables, respectively. Univariate analysis was performed to identify significant associations between PEDH and potential predictors, while a multivariate logistic regression model was applied to determine independent risk factors. A statistical significance level of 0.05 was used and statistical analysis was performed using IBM SPSS Statistics, version 20 (IBM Corp., Armonk, NY, USA).

\section{Results}

\section{Study population}

A total of 731 patients underwent esophagectomy between March 1997 and April 2018, among which 414 met the inclusion criteria (Table 1). The population included mostly males $(84 \%)$ diagnosed with adenocarcinoma $(71 \%)$ of the lower and junctional esophagus (81\%). Minimally invasive esophagectomy was performed in 311 patients $(75.1 \%)$, while $103(25 \%)$ underwent open surgery.

The median follow-up after esophagectomy was 16 (range, 6-177) months and overall survival was $68.4 \%$. Twenty-two cases $(5.3 \%)$ were diagnosed with $\mathrm{PEDH}$ within a mean period of 8 months after surgery. PEDH cases were submitted more often to neoadjuvant chemoradiotherapy $(\mathrm{P}=0.05)$ and showed higher rates of pathological complete response $(\mathrm{P}=0.02)$ and lymph node harvest $(\mathrm{P}=0.01)$. Esophagectomy technique and postoperative morbidity did not demonstrate a statistically significant association with PEDH.

\section{Surgical repair outcomes}

All patients developing PEDH were scheduled for surgical repair according to presenting symptoms (Table 2). PEDH presented more often with a left-side chest occupation (95\%) by the transverse colon (91\%). Early PEDH occurred in nine patients $(40.9 \%)$, and, at the time of diagnosis, patients typically presented with significant symptoms (77\%), including either respiratory discomfort $(36 \%)$ or indications of gastrointestinal obstruction (41\%). Sixteen $(73 \%)$ patients presented with acute clinical manifestation, while two patients experienced visceral 
Table 1 Study population demographics

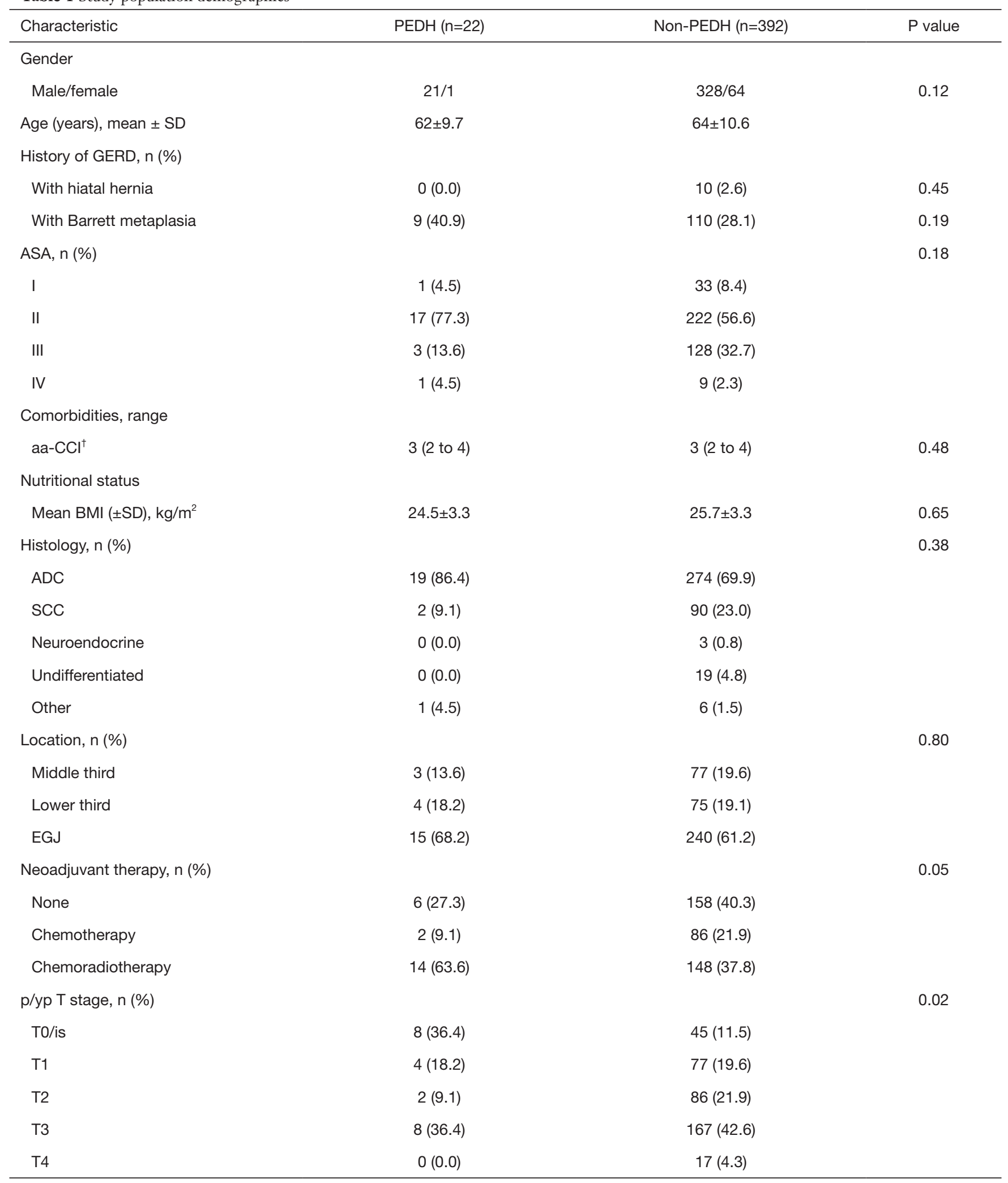

Table 1 (continued) 
Table 1 (continued)

\begin{tabular}{|c|c|c|c|}
\hline Characteristic & PEDH $(n=22)$ & Non-PEDH $(\mathrm{n}=392)$ & $P$ value \\
\hline No & $10(45.5)$ & $155(39.5)$ & \\
\hline $\mathrm{N}+$ & $12(54.5)$ & $237(60.5)$ & \\
\hline LN harvest ${ }^{\dagger}$, range & 39 (25.0 to 43.5$)$ & 32 (22.3 to 43.0$)$ & 0.01 \\
\hline Operative technique, n (\%) & & & 0.30 \\
\hline Open & $3(13.6)$ & $100(25.5)$ & \\
\hline Hybrid & $13(59.1)$ & $226(57.7)$ & \\
\hline Totally (MIL) & $6(27.3)$ & $66(16.8)$ & \\
\hline Reoperation & $3(13.6)$ & $31(7.39)$ & 0.41 \\
\hline Mean operative time $(\min )$, mean $\pm S D$ & $279 \pm 49$ & $271 \pm 68$ & 0.49 \\
\hline
\end{tabular}

${ }^{\dagger}$, median (inter quartile range); ${ }^{\ddagger}$, all complications consistent with grade $\geq 3 a$ according to Clavien-Dindo classification. PEDH, postesophagectomy diaphragmatic hernia; aa-CCl, age-adjusted Charlson Comorbidity Index.

ischaemia and perforation and underwent surgery within 24 hours. Postoperative morbidity was $22.7 \%$ and included exclusively cardiopulmonary complications, among which one death occurred due to respiratory failure. No early recurrences were found, and the median hospital stay after hernia repair was 6 (range, 2-95) days. Hernia repair was generally performed through a laparoscopic approach (77\%) with a conversion rate of $24 \%$. Moreover, cruroplasty was the repair method of choice (95\%), but surgical mesh was applied in one case to protect the diaphragmatic suture. After surgical repair, three recurrences (13.6\%) occurred over a median follow-up of 10.1 months and were successfully treated through laparoscopic technique.

\section{Risk factor analysis}

Univariate analysis was performed to determine significant associations between PEDH and potential perioperative predictors (17 items). The lymph node harvest threshold was identified by calculating the receiver operating characteristic curve and Youden Index, and a value of 33 nodes was adopted as the cut-off for the risk analysis (Table 3). Univariate analysis showed a statistically significant association between PEDH and pathological complete response [4.406 (1.752-11.084), $\mathrm{P}=0.001]$, preoperative chemoradiotherapy [2.885 (1.182-7.042), $\mathrm{P}=0.016]$, and lymph node harvest [2.894 (1.109-7.549), $\mathrm{P}=0.024]$. All predictors showing a significant association with $\mathrm{PEDH}$ entered the multivariate analysis, and pathological complete response $(\mathrm{P}=0.009)$ and lymph node harvest $(\mathrm{P}=0.026)$ were confirmed as independent risk factors.

\section{Discussion}

The occurrence rate of diaphragmatic hernia after esophageal surgery has been variably reported in the current literature, with an overall incidence ranging from $0.3 \%$ to $19 \%$. This measurable variety is related to the wide heterogeneity among study designs, including patient selection, type of esophageal resection, and length of postoperative follow-up (4). In order to minimize these limitations, this retrospective analysis has strictly selected patients from a prospective clinical database merging the experience of two high-volume esophageal cancer centers. In this study, the estimated incidence of diaphragmatic hernia after Ivor Lewis esophagectomy was 5.3\% within a median postoperative follow-up of 16 (range, 6-177) months, which is consistent with results reported by similar analyses $(2,7,12,18)$. After esophagectomy, PEDHs were identified in a mean period of $8( \pm 9.8)$ months, although the majority 
Table 2 Surgical outcomes after PEDH repair

\begin{tabular}{|c|c|}
\hline Characteristic & PEDH $(n=22)$ \\
\hline \multicolumn{2}{|l|}{ Primary tumor site, $\mathrm{n}(\%)$} \\
\hline Middle third & $3(13.6)$ \\
\hline Lower third & $4(18.2)$ \\
\hline S1 & $6(27.3)$ \\
\hline S2 & 9 (40.9) \\
\hline Mean interval to diagnosis $( \pm S D)$ & $8 \pm 9.8$ \\
\hline \multicolumn{2}{|l|}{ Setting } \\
\hline Urgent & $16(72.7)$ \\
\hline Elective & $6(27.3)$ \\
\hline \multicolumn{2}{|l|}{ PEDH contents, n (\%) } \\
\hline Colon & $15(68.2)$ \\
\hline Small bowel & $1(4.5)$ \\
\hline Both & $6(27.3)$ \\
\hline \multicolumn{2}{|l|}{ Surgical repair, n (\%) } \\
\hline Laparoscopic approach & $17(77.3)$ \\
\hline Conversion rate & $4(23.5)$ \\
\hline Cruroplasty & $21(95.5)$ \\
\hline Cruroplasty + mesh & $1(4.5)$ \\
\hline \multicolumn{2}{|l|}{ Postoperative morbidity, n (\%) } \\
\hline Atrial fibrillation & $1(4.5)$ \\
\hline Pneumonia & $1(4.5)$ \\
\hline Respiratory failure & $3(13.6)$ \\
\hline Early recurrence & $0(0.0)$ \\
\hline Median length of hospital stay (range) & 6 (2 to 95$)$ \\
\hline 30-day mortality, n (\%) & $1(4.5)$ \\
\hline Recurrence rate, n (\%) & $3(13.6)$ \\
\hline
\end{tabular}

$\mathrm{PEDH}$, post-esophagectomy diaphragmatic hernia.

of herniations occurred within the earliest 18 months (83\%). The clinical manifestations of PEDH typically included either respiratory or gastrointestinal symptoms. Sixteen patients $(73 \%)$ required urgent surgical treatment, among which two presented with complicated hernias (i.e., visceral ischaemia and perforation). Hernia repair was mainly performed through laparoscopic technique and, despite a conversion rate of $23.5 \%, 13$ patients $(59.0 \%)$ were successfully treated through minimally invasive surgery. Overall morbidity following the repairing procedure was $22.7 \%$, which mainly included severe respiratory complications, while one death occurred after repair of a complicated PEDH presenting with conduit necrosis due to gastrointestinal obstruction. In 2011, Price (3) highlighted that the clinical impact of diaphragmatic herniation following esophagectomy is related to the risk of visceral incarceration or strangulation and, thus, surgical repair should be recommended to prevent these life-threatening conditions $(4,19)$. The current study confirmed significant rates of acute presentation as well as postoperative morbidity and supported the recommendation of surgical repair. Additionally, repair procedure outcomes confirmed that laparoscopy is a feasible and effective repair technique in high-volume centers, especially in noncomplicated PEDH cases (7).

A risk factor analysis was performed and demonstrated that both pathological complete response and lymph node harvest are independent risk factors (0.009 and 0.026, respectively) for diaphragmatic herniation after Ivor Lewis esophagectomy for cancer. After a secondary analysis concerning possible confounding factors, we determined that pathological complete response showed a significant association with PEDH due to their longer overall survival after esophagectomy, which involves a higher likelihood of late diaphragmatic hernias. This analysis also identified lymph node harvest as an independent risk factor for PEDH, and the main reason for this association is the surgical demolition of esophageal cancer resections. Although the pathogenic mechanism of PEDH remains unclear, the loss of adhesions between the esophagogastric tract and the surrounding mediastinal and diaphragmatic structures is considered as a primary element leading to trans-hiatal herniation. The extent of lymphadenectomy is an expression of surgical demolition, and it has been associated with surgical complications after esophagectomy (20). The median number of removed nodes was substantial in this study population (32 nodes), and although a wide lymphadenectomy has been demonstrated to be an important prognostic factor for longterm outcomes, this parameter has been associated with higher rates of postoperative complications $(21,22)$. This risk factor analysis demonstrated that minimally invasive esophagectomy was not significantly associated with postoperative diaphragmatic herniation. However, previous studies demonstrated a significant association between keyhole surgery and the postoperative development of $\mathrm{PEDH}$. The primary reason would be the increase of intraabdominal pressure by the insufflation of carbon oxide that 
Table 3 Univariate and Multivariate logistic regression of risk factors

\begin{tabular}{|c|c|c|c|c|}
\hline Characteristic & \multicolumn{2}{|c|}{ Univariate analyses } & \multicolumn{2}{|c|}{ Multivariate analyses } \\
\hline Male & $4.329(0.572-32.741)$ & 0.122 & & \\
\hline Age $>60$ years & $0.830(0.346-1.989)$ & 0.675 & & \\
\hline $\mathrm{BMI}>25 \mathrm{~kg} / \mathrm{m}^{2}$ & $0.941(0.398-2.220)$ & 0.889 & & \\
\hline Neoadjuvant chemotherapy & $0.356(0.082-1.552)$ & 0.152 & & \\
\hline Neoadjuvant chemoradiotherapy & $2.885(1.182-7.042)$ & 0.016 & $2.403(0.951-6.072)$ & 0.064 \\
\hline Adenocarcinoma subtype & $2.107(0.698-6.355)$ & 0.177 & & \\
\hline Barrett metaplasia & $1.775(0.738-4.270)$ & 0.195 & & \\
\hline рT3-4 & $0.646(0.265-1.575)$ & 0.333 & & \\
\hline Lymph node count $>33$ & $2.894(1.109-7.549)$ & 0.024 & $3.029(1.140-8.049)$ & 0.026 \\
\hline OIL & $0.461(0.134-1.591)$ & 0.210 & & \\
\hline HIL & $1.061(0.443-2.541)$ & 0.894 & & \\
\hline MIL & $1.852(0.699-4.910)$ & 0.209 & & \\
\hline Severe morbidity $(C D \geq 3 a)^{\ddagger}$ & $0.747(0.286-1.954)$ & 0.551 & & \\
\hline Reoperation ${ }^{\ddagger}$ & $1.839(0.515-6.559)$ & 0.341 & & \\
\hline
\end{tabular}

${ }^{\ddagger}$, after esophagectomy. CD, Clavien-Dindo scoring system.

could lead to a progressive hiatal widening by the traction effect on tissues, while another explanation is the possible involvement of the lesser postoperative adhesions following minimally invasive surgery $(3,6,9,15)$. In addition, previous comparative studies revealed that minimally invasive esophagectomy could lead to equivalent surgical dissection and wider lymphadenectomy due to the intraoperative imaging magnification $(23,24)$. However, this analysis showed that open, hybrid, and total minimally invasive Ivor Lewis esophagectomy reported increasing occurrence rates of diaphragmatic hernia $(\mathrm{ORs}=0.461,1.061$, and 1.852, respectively) although the association between surgical technique and PEDH was not statistically significant. Despite these results, mechanisms triggering diaphragmatic herniation after esophagectomy remain unclear and too little evidence can support specific preventing measures. However, surgical prevention may include additional intraoperative procedures, such as the minimization of hiatal enlargement, repair of excessively large hiatus, anchoring of gastric conduit to the diaphragm, colopexy, and colonic omentectomy.

Although the primary limitation of this study was the retrospective design, data were prospectively collected in an individual clinical database and all patients underwent a standardized postoperative follow-up, which was equivalent between institutions and that contributed to reducing the selection bias. Another limitation was the wide range of postoperative follow-up, which was limited primarily by the number of advanced esophageal cancer cases and the overall survival. Therefore, further prospective and multicenter studies should be undertaken in order to perform stratified analyses involving more risk factors and patient characteristics.

\section{Conclusions}

In conclusion, the current study demonstrated an incidence rate of $5.3 \%$ of diaphragmatic hernia after Ivor Lewis esophagectomy for esophageal cancer. Risk factor analysis identified pathological complete response and lymph node 
harvest as independent risk factors although data could be affected by the group's overall survival. More often, PEDH involved the transverse colon and presented with either severe or mild symptoms. However, the risk of acute manifestation and the following significant postrepair morbidity support the recommendation for elective surgical treatment. Laparoscopic repair of non-complicated diaphragmatic hernia is feasible and effective in highvolume centers.

\section{Acknowledgments}

Funding: None.

\section{Footnote}

Reporting Checklist: The authors have completed the STROBE Statement reporting checklist. Available at http:// dx.doi.org/10.21037/jtd-20-1974

Data Sharing Statement: Available at http://dx.doi. org/10.21037/jtd-20-1974

Conflicts of Interest: All authors have completed the ICMJE uniform disclosure form (available at http://dx.doi. org/10.21037/jtd-20-1974). The authors have no conflicts of interest to declare.

Ethical Statement: The authors are accountable for all aspects of the work in ensuring that questions related to the accuracy or integrity of any part of the work are appropriately investigated and resolved. The present study was conducted in accordance with the Declaration of Helsinki (as revised in 2013). According to the national law regulating research ethics (resolution March 1, 2012, Gazzetta Ufficiale n.72 of March 26, 2012) and due to the retrospective design of the study, the ethical approval and informed consent have been waived since all analyzed data were collected as part of routine diagnosis and treatment, which were consistent with the current standard of care.

Open Access Statement: This is an Open Access article distributed in accordance with the Creative Commons Attribution-NonCommercial-NoDerivs 4.0 International License (CC BY-NC-ND 4.0), which permits the noncommercial replication and distribution of the article with the strict proviso that no changes or edits are made and the original work is properly cited (including links to both the formal publication through the relevant DOI and the license). See: https://creativecommons.org/licenses/by-nc-nd/4.0/.

\section{References}

1. Jamieson GG, Mathew G, Ludemann R, et al. Postoperative mortality following oesophagectomy and problems in reporting its rate. Br J Surg 2004;91:943-7.

2. Vallböhmer D, Hölscher AH, Herbold T, et al. Diaphragmatic hernia after conventional or laparoscopicassisted transthoracic esophagectomy. Ann Thorac Surg 2007;84:1847-52.

3. Price TN, Allen MS, Nichols FC 3rd, et al. Hiatal hernia after esophagectomy: analysis of 2,182 esophagectomies from a single institution. Ann Thorac Surg 2011;92:2041-5.

4. Oor JE, Wiezer MJ, Hazebroek EJ. Hiatal Hernia After Open versus Minimally Invasive Esophagectomy: a Systematic Review and Meta-analysis. Ann Surg Oncol 2016;23:2690-8.

5. Sutherland J, Banerii N, Morphew J, et al. Postoperative incidence of incarcerated hiatal hernia and its prevention after robotic transhiatal esophagectomy. Surg Endosc 2011;25:1526-30.

6. Benjamin G, Ashfaq A, Chang YH, et al. Diaphragmatic hernia post-minimally invasive esophagectomy: a discussion and review of literature. Hernia 2015;19:635-43.

7. Ulloa Severino B, Fuks D, Christidis C, et al. Laparoscopic repair of hiatal hernia after minimally invasive esophagectomy. Surg Endosc 2016;30:1068-72.

8. Gooszen JAH, Slaman AE, van Dieren S, et al. Incidence and Treatment of Symptomatic Diaphragmatic Hernia After Esophagectomy for Cancer. Ann Thorac Surg 2018;106:199-206.

9. Willer BL, Worrell SG, Fitzgibbons RJ Jr, et al. Incidence of diaphragmatic hernias following minimally invasive versus open transthoracic Ivor Lewis McKeown esophagectomy. Hernia 2012;16:185-90.

10. Kauppila JH, Xie S, Johar A, et al. Meta-analysis of healthrelated quality of life after minimally invasive versus open oesophagectomy for oesophageal cancer. Br J Surg 2017;104:1131-40.

11. Gottlieb-Vedi E, Kauppila JH, Malietzis G, et al. Longterm Survival in Esophageal Cancer After Minimally Invasive Compared to Open Esophagectomy: a Systematic Review and Meta-analysis. Ann Surg 2019;270:1005-17.

12. Biere SS, van Berge Henegouwen MI, Maas KW, et al. Minimally invasive versus open oesophagectomy for patients with oesophageal cancer: a multicentre, open-label, 
randomised controlled trial. Lancet 2012;379:1887-92.

13. Yibulayin W, Abulizi S, Lv H, et al. Minimally invasive oesophagectomy versus open esophagectomy for resectable esophageal cancer: a meta-analysis. World J Surg Oncol 2016;14:304.

14. Pasquali S, Yim G, Vohra RS, et al. Survival After Neoadjuvant and Adjuvant Treatments Compared to Surgery Alone for Resectable Esophageal Carcinoma: a Network Meta-analysis. Ann Surg 2017;265:481-91.

15. Fumagalli U, Rosati R, Caputo $M$, et al. Diaphragmatic acute massive herniation after laparoscopic gastroplasty for esophagectomy. Dis Esophagus 2006;19:40-3.

16. Dindo D, Demartines N, Clavien PA. Classification of surgical complications: a new proposal with evaluation in a cohort of 6336 patients and results of a survey. Ann Surg 2004;240:205-13.

17. Low DE, Alderson D, Cecconello I, et al. International Consensus on Standardization of Data Collection for Complications Associated With Esophagectomy: Esophagectomy Complications Consensus Group (ECCG). Ann Surg 2015;262:286-94.

18. Ganeshan DM, Correa AM, Bhosale P, et al. Diaphragmatic hernia after esophagectomy in 440 patients with long-term follow-up. Ann Thorac Surg

Cite this article as: Puccetti F, Cossu A, Parise P, Barbieri L, Elmore U, Carresi A, De Pascale S, Fumagalli Romario U, Rosati R. Diaphragmatic hernia after Ivor Lewis esophagectomy for cancer: a retrospective analysis of risk factors and post-repair outcomes. J Thorac Dis 2021;13(1):160-168. doi: 10.21037/jtd20-1974
2013;96:1138-45.

19. Kent MS, Luketich JD, Tsai W, et al. Revisional surgery after esophagectomy: an analysis of 43 patients. Ann Thorac Surg 2008;86:975-83; discussion 967-74.

20. Li H, Zhang ZR. Current status and future direction of lymph node dissection in radical surgery for esophageal cancer. J Thorac Dis 2019;11:S1678-82.

21. Greenstein AJ, Litle VR, Swanson SJ, et al. Effect of the number of lymph nodes sampled on postoperative survival of lymph node-negative esophageal cancer. Cancer 2008;112:1239-46.

22. Lagarde SM, Omloo JM, Ubbink DT, et al. Predictive factors associated with prolonged chest drain production after esophagectomy. Dis Esophagus 2007;20:24-8.

23. Klevebro F, Scandavini CM, Kamiya S, et al. Single center consecutive series cohort study of minimally invasive versus open resection for cancer in the esophagus or gastroesophageal junction. Dis Esophagus 2018. doi: 10.1093/dote/doy027.

24. Seesing MFJ, Gisbertz SS, Goense L, et al. A Propensity Score Matched Analysis of Open Versus Minimally Invasive Transthoracic Esophagectomy in the Netherlands. Ann Surg 2017;266:839-46. 